\title{
Occipital Nerve Stimulation for Medically Refractory Chronic Paroxysmal Hemicrania
}

\author{
${ }^{1}$ Sarah Miller MBBS, MRCP, ${ }^{1}$ Susie Lagrata BSc, ${ }^{2}$ Laurence Watkins FRCS, PhD and \\ ${ }^{1}$ Manjit Matharu FRCP, PhD \\ ${ }^{1}$ Headache Group, Institute of Neurology and The National Hospital for Neurology and \\ Neurosurgery, Queen Square, London, UK \\ ${ }^{2}$ Department of Neurosurgery, Institute of Neurology and The National Hospital for \\ Neurology and Neurosurgery, Queen Square, London, UK
}

Prepared For: Headache

Abstract: 174

Body: 1257

Total word count: 1431

Figures: 0

References: 7

\section{Corresponding Author:}

Dr MS Matharu

Senior Lecturer and Honorary Consultant Neurologist

Headache Group, Institute of Neurology and The National Hospital for Neurology and

Neurosurgery, Queen Square, London WC1N 3BG

Email: m.matharu@uclmail.net

Tel: $\quad+447595900535$

Fax: $\quad+447092120797$

\section{Running Title:}

ONS for $\mathrm{CPH}$

\section{Key Words:}

Paroxysmal hemicrania, Indometacin sensitive headache, Trigeminal autonomic cephalalgia, Intractable headache, Pregnancy, Neurostimulation, Occipital Nerve Stimulation 


\section{ABSTRACT:}

\section{Objective:}

To describe the outcome of a patient with refractory chronic paroxysmal hemicrania to occipital nerve stimulation.

\section{Background:}

Chronic paroxysmal hemicrania is a primary headache disorder exquisitely sensitive to indomethacin. In patients unable to tolerate indomethacin, the therapeutic options are limited. Occipital nerve stimulation is a promising therapy for other refractory headache conditions. We report the first patient with medically refractory chronic paroxysmal hemicrania treated with occipital nerve stimulation.

\section{Methods:}

Following implantation of the occipital nerve stimulator in 2006, the patient kept prospective headache diaries. Outcome was assessed by daily attack frequency.

\section{Results:}

After a follow-up of over 10-years, the patient reported a sustained efficacy of more than $50 \%$ reduction in attack frequency and was pain-free at final follow-up. The patient was able to stop indomethacin completely. The patient had three successful pregnancies during follow-up. One system revision was undertaken alongside an expected battery replacement to treat unequal paresthesia and pain over the electrodes.

\section{Conclusion:}


Occipital nerve stimulation may offer an effective long-term treatment for chronic paroxysmal hemicrania in patients where indomethacin cannot be tolerated.

Pg. 3 


\section{BACKGROUND}

Paroxysmal Hemicrania $(\mathrm{PH})$ is a rare primary headache disorder characterized by recurrent attacks of severe, strictly unilateral pain, focused around the orbital, supraorbital and temporal regions, and associated with autonomic features. Attacks can occur more than five times a day and last 2-30 minutes ${ }^{1}$. The disorder has an absolute response to indomethacin such that patients are rendered pain free by therapeutic doses. Between $65 \%$ and $88 \%$ of patients suffer from the chronic form of PH where the attacks occur for more than one year without remission, or with remission periods lasting less than one month ${ }^{1-3}$.

Over $30 \%$ of patients report dose-limiting side-effects with indomethacin and approximately $20 \%$ have to discontinue the drug due to tolerability problems ${ }^{4}$. Although several other drugs have been reported to have efficacy in $\mathrm{PH}$, none offer the same magnitude of response.

Occipital nerve stimulation (ONS) has been performed with positive outcomes in other primary headache conditions, including cluster headache. Given that cluster headache is a condition with similar clinical and pathophysiological features to $\mathrm{PH}$, we hypothesized that ONS may also be helpful to relieve medically refractory chronic paroxysmal hemicrania $(\mathrm{CPH})$. We report on the first patient with severe medically refractory $\mathrm{CPH}$ to undergo ONS.

\section{CASE REPORT}

A 26-year-old woman had a five-year history of recurrent attacks of excruciating strictly unilateral "sharp", "electric shock-like" episodes of pain centered over the parietal and temporal regions radiating to the forehead and neck. She reported daily attacks with no remission period since onset. The attacks occurred predominately on the right side of the head (90\% of attacks) although she had occasional unilateral side-variable attacks involving 
the left side . Attacks would occur between 6-8 times daily with at least three of these at night. Attacks lasted an average of 15 minutes (ranging from 5-60 minutes) and were associated with ipsilateral conjunctival injection, ptosis, nasal blockage, rhinorrhea, facial sweating, photophobia and phonophobia. She also complained of nausea and restlessness with attacks. Triggers for the attacks included the smell of volatile substances (perfume, bleach), sleep deprivation and ingestion of cheese and chocolate. The patient had complete resolution of pain with an oral indomethacin test (taken to a maximum of $75 \mathrm{mg}$ tds) and a diagnosis of $\mathrm{CPH}$ was made. She was taking $150 \mathrm{mg}$ daily of indomethacin but was suffering severe gastric side effects despite proton-pump inhibitors.

The patient had a history of co-existent episodic migraine with aura that had started at the age of 14 years. This was very different to her $\mathrm{CPH}$ and was a moderately severe bilateral throbbing headache centered over the forehead. Her migraine was associated with a visual aura, nausea, photophobia, phonophobia, osmophobia and motion sensitivity. She suffered around four episodes of migraine a year each lasting between 12-24 hours. These episodes responded to paracetamol.

Other medical history included previous thyrotoxicosis treated successfully with carbimazole between the ages of 16-20 years. She had since been euthyroid without treatment. The patient reported a similar indomethacin-responsive headache disorder in her mother. The patient was a junior doctor and had managed to continue working despite her headache disorder.

She had tried multiple preventative medications including verapamil, rofecoxib, celecoxib, etoricoxib, methysergide, melatonin, topiramate, pizotifen and amitriptyline. Greater occipital nerve blocks had proven ineffective. Given the intractable nature of her condition she was referred for a bilateral greater occipital nerve stimulator. 
A bilateral greater occipital nerve stimulator was implanted in September 2006 using quad electrodes and a single IPG (Medtronic Prime Advanced; Medtronic Inc., Minneapolis, MN, USA). Bilateral leads were employed to prevent side-shifting of attacks ${ }^{5}$. This was placed on continuous bilateral stimulation with a pulse width of $450 \mu \mathrm{s}$ and a frequency of $70 \mathrm{~Hz}$. Over the course of treatment amplitude was altered to maintain constant occipital paraesthesiae and ranged from $0.3-0.7 \mathrm{~V}$. Further details of occipital nerve stimulator implantation and programming techniques can be found in detail elsewhere ${ }^{6}$.

Within two weeks of implant the patient reported attacks occurring at 1-2 times a day with no change in severity or duration. After one month, attacks were occurring only once a week and she was able to completely stop Indomethacin. Initial problems with painful or unequal stimulation on the left hand side were only partly solved with re-programming of the device. The patient also complained of constant discomfort along the track of the electrodes and neck stiffness.

Efficacy was maintained until late 2008 when the patient noted intermittent paraesthesiae and a gradual increase in attack frequency. The battery was found to be at the end of life and due to the complaint of unequal paresthesia and pain over the electrode tracks a decision was made to replace the whole system with octad electrodes and a rechargeable battery. This was undertaken in October 2009 without complication. Unfortunately, following the system replacement it took a number of months to regain the same level of efficacy as previously experienced. In October 2011 attack frequency was back to once a week but she also reported a significant reduction in attack duration down to 5-minutes. The patient has remained well controlled with ONS with only occasional breakthrough of attacks in 2013 and 2015. The episode in 2013 was associated with a sudden increase following a road traffic accident and loss of the stimulator control handset in the accident meaning that she could not adjust her stimulation levels herself. The ONS system was found to be working well with no 
lead fracture or displacement following the accident and the symptoms resolved within a few months of reprogramming. Another transient increase in attack frequency was seen in early 2015 triggered by stress. The patient reported up to 10-20 attacks a day of severe pain lasting 2-5 minutes each. Due to the severity of the bout she was advised to restart Indomethacin and the device was reprogrammed. By mid-2015 she reported her attacks had improved to 3 attacks daily lasting on average 4 -minutes and she had been able to stop Indomethacin. The ONS was reprogrammed and the patient remained pain free until review in early-2017.

Since the ONS implant, the patient has had three successful pregnancies with no issues from the implant occurring in any. During all pregnancies she was able to control her $\mathrm{PH}$ well and not use Indomethacin. She did report an increase in her episodic migraines during her second pregnancy but these settled after delivery.

\section{DISCUSSION}

This article reports the first patient to receive bilateral ONS for CPH. Similar sustained efficacy has been noted in chronic cluster headache and hemicrania continua, two conditions sharing both clinical and pathophysiological features with $\mathrm{CPH}^{3,7}$. The patient reported a delay of around one month to initial improvement and nearly 8-12 months to regain that level of improvement following battery depletion and ONS revision. Similarly, she reported a gradual increase in attack frequency as the battery failed over a number of months. These time delays have been consistently reported in the series of other headache conditions and argue against a placebo response as the cause of improvement.

The long-term control of the attacks was interspersed with occasional bouts of attacks. These were controlled with reprogramming of the ONS device. This observation suggests that ONS 
is modifying the neural network involved in pain generation but does not modify the natural history of the condition with periods of remissions and exacerbations. It is important that patients undergoing the treatment are counseled that a resurgence of attacks does not mean treatment failure but is a normal and expected observation.

We conclude that ONS may offer an effective long-term treatment for medically refractory $\mathrm{CPH}$ in patients where indomethacin cannot be tolerated. 


\section{CONSENT FOR PUBLICATION:}

The subject has read and consented for the publication of this case report.

\section{AUTHOR CONTRIBUTIONS:}

SM: recruitment of subject, analysis and interpretation of data, drafting and revision of manuscript.

SL: collection of data

LW: performed surgery and manuscript revision.

MSM: paper concept, recruitment of subjects, interpretation of data and manuscript revision.

\section{DISCLOSURES:}

SM has received educational and travel grants from St Jude Medical and Medtronic. SL reports no disclosures. LW has served on advisory boards for St Jude Medical and Medtronic. MSM serves on the advisory board for Allergan, St Jude Medical and Medtronic and has received payment for the development of educational presentations from Allergan, Merck Sharpe and Dohme Ltd, Medtronic and electroCore.

\section{COMPETING INTERESTS:}

SM has received educational and travel grants from St Jude Medical and Medtronic. SL has no competing interests. LW declares no competing interests. MSM serves on the advisory board for Allergan, St Jude Medical and Medtronic and has received payment for the development of educational presentations from Allergan, Merck Sharpe and Dohme Ltd, Medtronic and electroCore. 


\section{STUDY FUNDING:}

Nil

\section{ACKNOWLEDGEMENTS:}

We would like to thank our Headache Specialist Nurses for their help with completion of the clinical database and management of the patients. We also thank the patient and her family for their help with this project. 


\section{REFERENCES:}

1. Headache Classification Subcommittee of The International Headache Society. The International Classification of Headache Disorders 3rd edition (beta version). Cephalalgia. 2013; 33: 629-808.

2. Cittadini E, Matharu MS and Goadsby PJ. Paroxysmal hemicrania: a prospective clinical study of 31 cases. Brain. 2008; 131: 1142-55.

3. VanderPluym J. Indomethacin-responsive headaches. Curr Neurol Neurosci Rep. 2015; 15 : 516.

4. Dodick DW. Indomethacin-responsive headache syndromes. Current pain and headache reports. 2004; 8: 19-26.

5. Magis D, Gerardy PY, Remacle JM and Schoenen J. Sustained effectiveness of occipital nerve stimulation in drug-resistant chronic cluster headache. Headache. 2011; 51: 1191-201.

6. Miller S, Watkins L and Matharu M. Long-term outcomes of occipital nerve stimulation for chronic migraine: a cohort of 53 patients. J Headache Pain. 2016; 17: 68.

7. Magis D and Schoenen J. Advances and challenges in neurostimulation for headaches. Lancet Neurol. 2012; 11: 708-19. 